\title{
FRAÇÕES FOSFATADAS EM MUDAS DE Eucalyptus ${ }^{(1)}$
}

\author{
A. E. FURTINI NETO(2), N. F. BARROS(3), \\ R. F. NOVAIS ${ }^{(3)} \&$ M. F. G. OLIVEIRA(4)
}

\begin{abstract}
RESUMO
Com o objetivo de avaliar os teores e o efeito do tempo de omissão de fósforo nas frações de $P$ em diferentes espécies de eucali pto, instal ou-se um experimento em condições controladas no Departamento de Solos da Universidade Federal de Viçosa - UFV, no período de fevereiro a julho de 1993. Avaliou-se, em câmara de crescimento, o comportamento das frações fosfatadas em mudas das segui ntes espécies de eucalipto: E. clöeziana, E. grandis, E. pellita, E. pi lularise E. urophylla, quando submetidas à omissão de $P$. Foi fornecida às plantas uma sol ução nutritiva com el evado teor de $\mathrm{P}\left(8 \mathrm{mg} \mathrm{L}^{-1}\right.$ de $\left.\mathrm{P}\right)$ por vinte e quatro horas. Após esse período, eli minou-se o $\mathbf{P}$ da solução nutritiva e anal isaram-se as frações de $P$ total solúvel em ácido (Pts), fósforo inorgânico (Pi) e fósforo orgânico (Po), nos tempos de 0 , 10 e 20 dias após a omissão do nutriente. Os compartimentos analisados foram as partes apicais e basais de folhas e raízes. A omissão do $P$ da solução nutritiva causou queda no conteúdo de Pts e Pi em todas as espécies, embora em diferente magnitude. E. clöeziana apresentou o maior conteúdo de Pts. Com o aumento do tempo de omissão, as espécies de eucali pto apresentaram redução no percentual de partici pação do $\mathrm{Pi}$ em relação ao $\mathrm{Pts}$, seguido de maior partici pação relativa do Po nos diversos compartimentos analisados. E. pellita e E. grandis apresentaram um padrão mais homogêneo de comportamento das frações fosfatadas com o tempo de omissão, quando comparadas às outras espécies. Entretanto, essas espécies apresentaram baixos teores de Pi já aos 10 dias de omissão de $\mathrm{P}$, indicativo de um baixo "poder tampão interno de $\mathrm{Pi}$ " em relação às outras espécies avaliadas.
\end{abstract}

Termos de indexação: frações fosfatadas, eucalipto.

(1) Parte da Tese de Doutorado do primeiro autor, apresentada ao Departamento de Solos da Universidade Federal de Viçosa (UFV), Viçosa (MG). Recebido para publicação em outubro de 1996 e aprovado em março de 1998.

(2) Professor do Departamento de Ciência do Solo, Universidade Federal de Lavras. CEP 37200-000 Lavras (MG).

(3) Professor do Departamento de Solos, Universidade Federal de Viçosa. CEP 36571-000 Viçosa (MG).

(4) Bolsista da EMBRAPA - Centro Nacional de Pesquisa de Milho e Sorgo. Caixa Postal 151, CEP 35701-970 Sete Lagoas (MG). 


\title{
SUMMARY:PHOSPHATE FRACTIONS IN EUCALYPT SEEDLINGS
}

\begin{abstract}
This experiment was carried out in the Department of Soil Science of the Federal University of Viçosa, Minas Gerais State, Brazil, with theobjective of evaluating the effect of $\mathrm{P}$ concentration and the duration of the $\mathrm{P}$-omission period on three different fractions of $\mathrm{P}$ in Eucalyptus seedlings: i) total soluble P (TSP); ii) inorganic P (IP); and iii) organic P $(\mathrm{OP})$. A solution containing high amount of phosphorus $\left(8 \mathrm{mg} \mathrm{L}^{-1}\right.$ of $P$ ) was added to five species of Eucalyptus (E. döeziana, E. grandis, E. pellita, E. pilularis and E. urophylla) during 24 hours. After this period, $\mathrm{P}$ was totally el iminated from the nutrient sol ution, and the fractions of TSP, IP and OP wereanalyzed at 0,10 , and 20 days after theomi ssion of the nutrient. Basal and apical leaves, as well as the extremity and basal portion of the root system, were analyzed for the different $P$ fractions. Phosphate omission from the nutrient solution decreased the TSP and IP content in all species, though the magnitude of this decreasevaried among species. E ucalyptus döeziana presented thehighest TSP content. As the P-omission period increased, the eucalypt species showed reduction in the percentage of partici pation of IP related to TSP, fol lowed by a greater relative participation of OP in the different plant compartments. As the P-omission period increased, E. pellita and E. grandis showed a more gradual decrease in the IP/ TSP ratio compared with the other species. However, E. pellita and $\mathrm{E}$. grandis had smaller IP amounts at 10 days of $\mathrm{P}$ omission, which indicates a lower "IP internal buffer capacity" compared with the other eucalypt species.
\end{abstract}

Index terms: phosphatefractions, eucalypt.

\section{INTRODUÇÃO}

Quando submetidos à mesma condição de fertilidade do solo, espécies e genótipos têm apresentado comportamento diferencial com relação à nutrição e crescimento. As diferenças nutricionais observadas entre plantas, rel acionadas com absorção, transporte e utilização de nutrientes, estão sob controle genético (E pstein \& J effries, 1964). De acordo com Gerloff \& Gabelman (1983), é de particular importância que se consi derem a al ocação interna do nutriente esua utilização no metabolismo e crescimento.

No caso particular do fósforo, as diferenças na absorção do nutriente têm sido atribuídas, entre outras, à diferença na modulação de seus mecanismos morfológicos efisiológicos, podendo-secitar, entre os últimos, diferenças na retenção e movimentação do nutriente absorvido e sua conseqüente liberação no xilema (Bieleski, 1973; Bieleski \& Ferguson, 1983; Vose, 1987).

A planta apresenta quatro compartimentos entre os quais ofósforo se movimenta: o apoplasto externo, em contato com a sol ução do sol o; o apoplasto interno, em contato com o corpo principal da planta; o citoplasma ou espaço metaból ico, e o espaço vacuolar ou não-metabólico, delimitado pelo tonoplasto (Bieleski \& Ferguson, 1983). É de supor, portanto, que o comportamento diferencial de plantas se deva não só à ocorrência de variações no transporte a longa distância e aos drenos preferenciais para o fósforo sob condições de estresse, mas também às diferenças no acúmulo eliberação de fósforo em nível vacuolar e na proporção relativa das frações metabólicas e não-metabólicas do nutriente.
Apesar de os mecanismos envolvidos ainda não serem total mente explicados, a dinâmica das frações fosfatadas está relacionada com a atividade de fosfatases. À medida que diminui ofornecimento deP paras as plantas, ocorre um aumento da atividade dessas enzimas (Bieleski \& Ferguson, 1983).

Estudos com diversas espécies têm mostrado que a concentração de fósforo inorgânico $(\mathrm{Pi})$ no citoplasma éregulada pel o seu conteúdo no vacúolo, ocorrendo mudanças acentuadas no conteúdo de Pi das células, quase exclusivamenteno" "pool"vacuolar, não havendo alterações significativas no "pool" citoplasmático (fósforo orgânico - Po) (Biel eski, 1973, Chapin et al., 1982; Lee \& Ratcliffe, 1983). O Pi vacuolar tem função de reserva e, se a quantidade de fósforo absorvida for menor que a sua demanda pela célula, el e retorna ao citoplasma, atendendo à exigência metabólica da célula (Biel eski \& Ferguson, 1983). Às vezes, esse retorno pode ser de pequena magnitude (Bieleski, 1973). Se o ritmo de crescimento for lento e a demanda de fósforo pequena, um aporte el evado do nutriente à planta resultaria em grande acúmulo de Pi vacuolar (Bieleski \& Ferguson, 1983). É de se supor que, embora possam apresentar a mesma tendência de comportamento, espécies distintas apresentem diferenças na dinâmica interna do nutriente.

Neste contexto, o acompanhamento dos teores de Pi, Po e fósforo total solúvel em ácido (Pts) (Fabres, 1986), em distintas espécies de eucal ipto, permitiria avaliar possíveis diferenças dessas espécies em armazenar Pi nos vacúolos, liberando-o, quando necessário, para ocitoplasma, além desua capaci dade detranslocar onutriente de regi ões menos ativas para regiões mais ativas metabolicamente. Tal 
comportamento poderia determinar diferenças na eficiência das espécies na utilização do fósforo.

O objetivo deste trabal ho foi avaliar os teores e o efeito do tempo de omissão de fósforo nas frações deste nutriente em mudas de diferentes espécies de eucalipto.

\section{MATERIAL E MÉTODOS}

O experimento foi desenvolvido em condições controladas, no Departamento de Solos da Universidade Federal de Viçosa - UFV, no período de fevereiro a julho de 1993. Sementes de cinco espécies de eucalipto, Eucalyptus clöeziana, E. grandis, E. pellita, E. pilularis eE. urophylla, foram colocadas para germinar em leito de areia lavada, onde as plântulas permaneceram até atingir aproximadamente cinco centímetros de altura. Em seguida, cerca de 30 mudas de cada espécie foram transferidas para bacias plásticas de 30 litros, onde a solução continha os nutrientes em metade da concentração ( $1 / 2$ força) recomendada por Clark (1975). Foi fornecido arejamento constante, e o pH da solução foi ajustado diariamente para 6,0, com a utilização de $\mathrm{HCl}$ e, ou, $\mathrm{NaOH}$. A solução nutritiva foi trocada a cada 10 dias e, após a terceira troca (30 dias), as plantas receberam solução com os nutrientes na concentração recomendada por Clark (1975). Durante esse período, as plantas permaneceram em casa de vegetação.
Ao atingirem aproximadamente $25 \mathrm{~cm}$ dealtura, seis plantas de cada espécie foram selecionadas quanto à uniformidade da parte aérea e sistema radicular etransferidas para câmara de crescimento, onde permaneceram por oito dias na mesma solução nutritiva em que vinham sendo cultivadas, para aclimatação ao novo ambiente. Duranteesse período e no decorrer do experimento, a câmara teve sua temperatura controlada em torno de $27^{\circ} \mathrm{C}$, a intensidade luminosa aproximada de 7.500 lux, à altura das folhas superiores, e a umidade relativa do ar em torno de $50 \%$.

Decorrido o período de aclimatação, a solução nutritiva das bacias foi renovada, tendo sido fornecida uma solução (Clark, 1975) com el evadoteor de fósforo, na tentativa de el evar ao máximo o teor dePi no vacúolo (Bieleski, 1973; Bieleski \& Ferguson, 1983). O teor de fósforo da solução nutritiva nessa fasefoi de $8 \mathrm{mg} \mathrm{L}$-1 deP. A pós $24 \mathrm{~h}$, a soluçãofoi trocada, eliminando-se o P de sua composição.

No momento de submeter as plantas à solução sem $\mathrm{P}$, for am coletadas duas plantas de cada espécie, permanecendo as restantes nas bacias plásticas. $\mathrm{O}$ material vegetal foi separado em fol has superiores e inferiores, e as raízes, separadas em partes apical e basal do sistema radicular. As amostras foram acondicionadas em ácido perclórico 0,2 mol L-1 e armazenadas em freezer, para posterior determinação dos teores de $\mathrm{P}$ inorgânico solúvel em ácido (Pi), $\mathrm{P}$ total solúvel (Pts) e, por diferença, o teor de $\mathrm{P}$ orgânico (Po), conforme método descrito por Smillie \& Krotkov (1960) e Hogue et al. (1970) modificado (Martinez, 1992), (Figura 1).

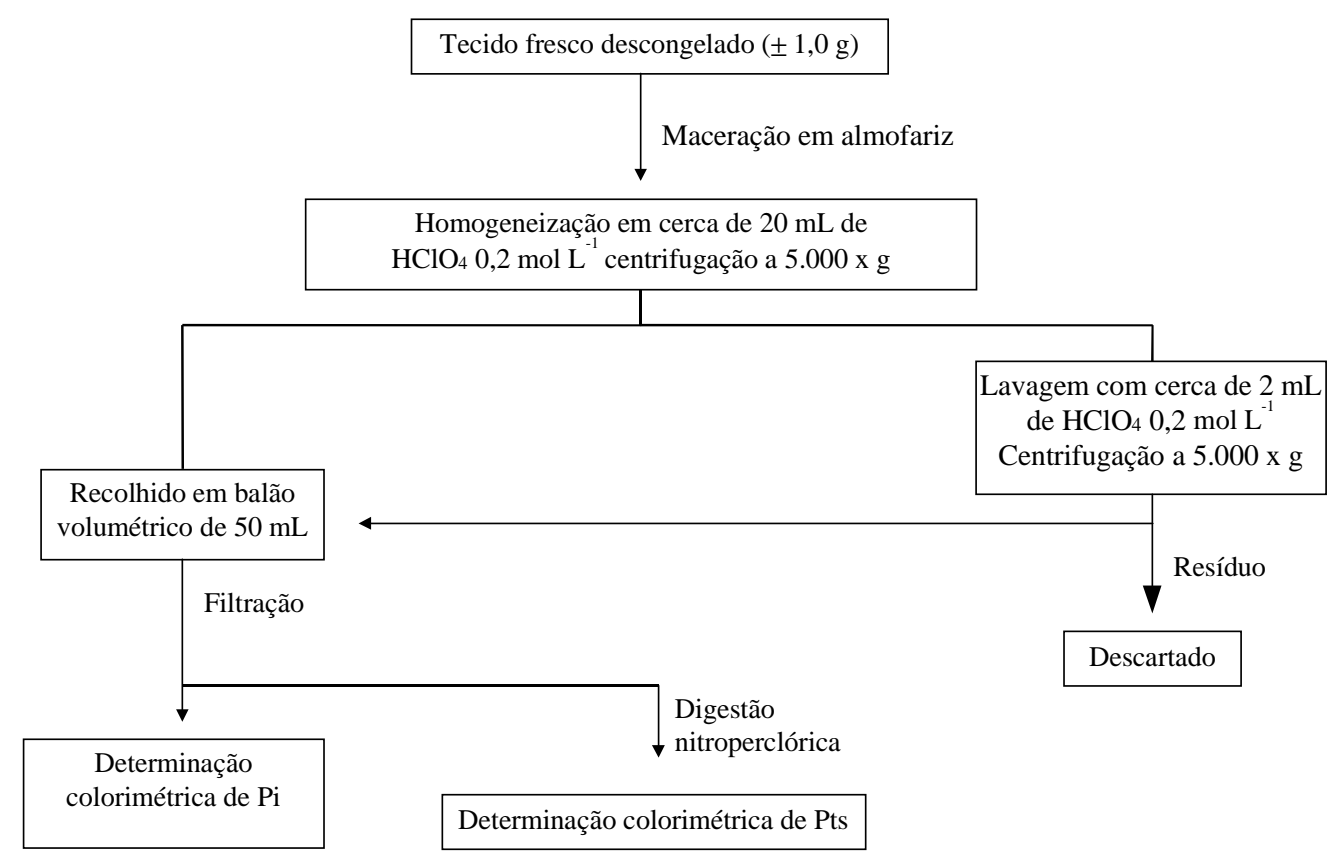

Figura 1. Fluxograma do método empregado no fracionamento de $P$ (Martinez, 1992). 
Dez evinte dias após a transferência das plantas para a solução sem fósforo, foram col etadas outras duas plantas de cada espécie em cada tempo, adotando-se o mesmo método de separação e acondicionamento do material vegetal.

$\mathrm{Na}$ determinação do fósforo inorgânico, em virtude da variação na coloração dos extratos após maceração e lavagem com $\mathrm{HClO}_{4}$ 0,2 mol L-1, decorrente de diferenças do material vegetal das espécies, foram efetuadas duas leituras, sem e com a adição do mol ibdato de amônio (Braga \& Defelipo, 1974). A leitura do extrato sem adição de molibdato de amônio serviu como "branco" e foi subtraída da leitura em que o molibdato foi adicionado.

Para comparar as frações fosfatadas das espécies, em função dos tempos de omissão, os resultados foram submetidos à anál ise de variância, eas médias foram comparadas pel o teste de Tukey (5\%).

\section{RESULTADOS E DISCUSSÃO}

\section{Frações fosfatadas}

A omissão do fósforo da sol ução nutritiva causou queda no conteúdo dePts ePi em praticamentetodas as espécies (Quadro 1). A única espécie que apresentou padrão diferente foi o $\mathrm{E}$. clöeziana, que, nos primeiros dez dias, manteve o conteúdo de Pts mais el evado.
Apesar da queda geral nos valores de Pts, com o avanço do tempo de omissão de $P$ na solução nutritiva, as espécies de eucalipto estudadas apresentaram variações nos intervalos de omissão considerados. E. clöeziana apresentou conteúdo de Pts mais el evado comparativamente às demais, em todo período experimental. Sob alta disponibilidade de $P$, essa espécie acumula grande quantidade do nutriente (Furtini Neto et al., 1996), sendo tais resultados coerentes com o elevado conteúdo de fósforo encontrado nessas plantas, quando cultivadas em sol o com el evadas doses de P. Esse al to conteúdo de Pts resulta da menor taxa de crescimento e da taxa rel ativamente alta de absorção de $\mathrm{P}$.

Antes de seefetuar a omissão defósforo (tempo 0), E. pilularis compartimentalizou mais de $75 \%$ do Pts na forma de $\mathrm{Pi}$, enquanto, nas demais espécies, esta partici paçãovariou de 46 a 50\%. A queda do conteúdo dePi deE. pilularis com o tempo de omissão de P foi mais suave, se comparada com a de outras espécies deste estudo. E. urophylla mostrou redução no conteúdo de Pi similar à do E. clöeziana, enquanto as outras espécies formaram um grupo distinto nesse aspecto (Quadro 1).

Enquanto E. clöeziana e E. urophylla apresentaram queda de 53 e $45 \%$, respectivamente, no conteúdo dePts após 20 dias de omissão, E. pel lita e E. pilularis mostraram redução aproximada de $11-12 \%$ no conteúdo de Pts. A queda no conteúdo de Pts das espécies estudadas foi devida, principalmente, às reduções nos níveis internos de Pi. Uma vez que

Quadro 1. Conteúdo de fósforo total solúvel em ácido (Pts), fósforo inorgânico (Pi) e fósforo orgânico (Po) em plantas (somatório do conteúdo de folhas superiores, inferiores e raízes da ponta e da base do sistema radicular) de cinco espécies de eucalipto após a omissão do nutriente (média de duas repetições)

\begin{tabular}{|c|c|c|c|c|c|c|}
\hline $\begin{array}{c}\text { Tempo de } \\
\text { omissão de P }\end{array}$ & $\begin{array}{l}\text { Fração } \\
\text { fosfatada }\end{array}$ & E. clöeziana & E.grandis & E. pellita & E. pilularis & E. urophylla \\
\hline Dia & & 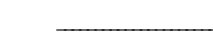 & 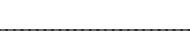 & $-\mu \mathrm{g} / \mathrm{planta}$ & 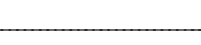 & - \\
\hline 0 & $\begin{array}{l}\text { Pts } \\
\mathrm{Pi} \\
\mathrm{Po}\end{array}$ & $\begin{array}{l}320,87 \\
150,70 \\
170,17\end{array}$ & $\begin{array}{r}105,53 \\
46,28 \\
59,24\end{array}$ & $\begin{array}{r}125,63 \\
57,85 \\
67,78\end{array}$ & $\begin{array}{r}149,20 \\
112,67 \\
36,52\end{array}$ & $\begin{array}{r}136,04 \\
68,18 \\
67,86\end{array}$ \\
\hline 10 & $\begin{array}{l}\text { Pts } \\
\mathrm{Pi} \\
\mathrm{Po}\end{array}$ & $\begin{array}{r}292,49 \\
77,59 \\
214,90\end{array}$ & $\begin{array}{r}177,14 \\
51,49 \\
125,65\end{array}$ & $\begin{array}{r}178,18 \\
32,37 \\
145,80\end{array}$ & $\begin{array}{r}190,05 \\
81,61 \\
108,44\end{array}$ & $\begin{array}{r}132,16 \\
25,56 \\
106,60\end{array}$ \\
\hline 20 & $\begin{array}{l}\text { Pts } \\
\mathrm{Pi} \\
\mathrm{Po}\end{array}$ & $\begin{array}{r}150,12 \\
36,95 \\
113,17\end{array}$ & $\begin{array}{r}106,35 \\
25,42 \\
80,93\end{array}$ & $\begin{array}{r}111,65 \\
29,10 \\
82,54\end{array}$ & $\begin{array}{r}131,25 \\
42,38 \\
88,86\end{array}$ & $\begin{array}{l}74,47 \\
15,14 \\
59,33\end{array}$ \\
\hline $\begin{array}{c}\text { \% de redução } \\
0-20 \text { dias }\end{array}$ & $\begin{array}{l}\text { Pts } \\
\mathrm{Pi}\end{array}$ & $\begin{array}{l}53 \\
75\end{array}$ & 45 & $\begin{array}{l}11 \\
50\end{array}$ & $\begin{array}{l}12 \\
62\end{array}$ & $\begin{array}{l}45 \\
78\end{array}$ \\
\hline
\end{tabular}

DMS (5\%) Espécie x Tempo: Pts = 36,35; Pi =43,45; Po = 70,09. CV (\%): 6,56; 21,94 e 19,66 respectivamente. 
a reserva fosfatada da planta éo Pi (Bieleski, 1973), a queda no seu conteúdo era esperada. Após 20 dias de omissão, E. clöeziana foi a espécie que ainda mostrou el evado conteúdo de Pts. Reduções no Pi em espécies sob deficiência de $P$ também foram observadas por Bieleski \& Ferguson (1983).

Em termos práticos, tais resultados indicam que, depois debemsupridas deP, E. döeziana eE. urophylla suportariam por mais tempo uma interrupção no fornecimento do nutriente, do que as demais, antes de paralisar seu crescimento. Entretanto, prováveis diferenças na eficiência de utilização de fósforo impedem que se considerem somente tais aspectos no desempenho das espécies com relação à produção de biomassa (Furtini N eto et al., 1996).

De acordo com Barros et al. (1990), al gumas procedências de $\mathrm{E}$. clöeziana devem apresentar em seu habitat natural uma absorção rápida de nutrientes do sol o e conseqüente armazenamento interno, causado por períodos de maior disponibilidade de água nas regiões de origem, para posterior metabolismo durante os períodos mais secos. Essa inferência é confirmada pelos resultados aqui obtidos.

Com a redução da concentração iônica externa, a primeira resposta da planta seria um declínio na concentração iônica do citoplasma que, por sua vez, seria o sinal, para que os transportadores em nível de plasmalema e tonoplasto restaurassem essa concentração, quer por aumentos na taxa de "turnover", quer por síntese induzida de moléculas de carregador. Se a concentração iônica externa for muito baixa, e os ajustes da plasmalema forem ineficazes, a mobilização deíons no vacúolo, por meio de alteração na atividade e, ou, concentração dos transportadores em nível de tonoplasto, será a fonte primária deíons para manter a concentraçãoiônica do citoplasma (Glass \& Siddiqi, 1984).

É provável que diferenças genéticas entre espécies resultem em diferentes graus de ajuste metabólico. Em última análise, diferenças nesses ajustes poderão resultar em vantagem ou desvantagem adaptativa às condições desfavoráveis de nutrição (Bieleski \& Ferguson, 1983). A maior produção de biomassa de E. urophylla, E. grandise principalmenteE. pellita, quando cultivadas em solo (Furtini Neto et al., 1996), sob várias doses de fósforo, provavelmente possa ser devida à eficiência nos ajustes metabólicos dessas espécies.

O conteúdo de Po das espécies de eucal ipto sofreu pequena el evação, atingindo valores máximos 10 dias após a omissão, para depois declinar. Tal comportamento, provavelmente, se deva ao fato de a demanda por Pocontinuar a elevar-se para atender às exigências de crescimento, sendo essa fração mantida à custa do Pi vacuolar liberado. Martinez (1992) também observou ligeiro aumento dos teores de Po e posterior queda, após período de omissão do nutriente em distintas variedades de soja.

\section{Frações fosfatadas nos diferentes compartimentos da planta}

Todas as espécies de eucalipto estudadas apresentaram redução do percentual de partici pação do Pi em relação ao Pts com o aumento do tempo de omissão, seguida de maior partici pação relativa do Po em relação ao Pts nos diversos compartimentos da planta (Quadros 2 e 3). Uma vez que o fornecimento de $\mathrm{P}$ foi interrompido, ajustes metabólicos nas espécies promoveram a liberação do Pi-vacuolar para o suprimento das necessidades de crescimento da planta, conforme sugerido por Bieleski \& Ferguson (1983).

A magnitude de decréscimo da participação do $\mathrm{Pi}$ e Pts é distinta entre as espécies de eucalipto estudadas. No caso de E. clöeziana e E. urophylla, a partici pação do Pi queé de 56 e $53 \%$ nas fol has novas atinge 15 e $16 \%$, respectivamente, no final do período de omissão, enquanto, nas demais espécies, a participação final do $\mathrm{Pi}$ em relação ao Pts é de aproximadamente 25\% (Quadro 2). Considerando o teor inicial dePi nas fol has apicais, E. clöeziana eE. urophylla apresentaram as maiores quedas na participação relativa aos 10 dias de omissão, atingindo 76 e $73 \%$, respectivamente, enquanto que para E. pilularis a queda foi de somente $55 \%$ da participação inicial (tempo 0). E. grandis eE. pellita formaram um grupo intermediário.

A maior percentagem de Pi nas fol has basais foi observada em E. pilularis (96\%), diminuindo linearmente com o tempo de omissão do fornecimento de $P$, atingindo valores relativos semel hante aos das demais espécies. E. grandis, E. pellita eE. urophylla apresentaram menores quedas na participação relativa ao $\mathrm{Pi}$ em relação à participação inicial, embora, aos 10 dias de omissão, a queda já fosse bem acentuada (Quadro 2). N ota-se que, aos 20 dias de omissão de P, E. clöeziana apresentou teores de Pts e Po bastante elevados, comparados aos das demais espécies.

Notocanteàs raízes apicais (Quadro 3), observouse acentuada queda na participação relativa de $\mathrm{Pi}$ do E. urophylla e E. pellita após 10 dias de omissão deP, atingindo 89 e $67 \%$, respectivamente. $\mathrm{E}$. grandis manteve a participação relativa mais ou menos constante até 10 dias de omissão, mas atingiu uma redução de $85 \%$ aos 20 dias. Nas raízes apicais, E. urophylla, E. grandis e E. pellita foram as espécies com os mais baixos teores de $\mathrm{Pi}$ no tempo final de omissão de P.

Nas raízes basais, éinteressante observar queE. grandis, E. pellita e E. urophylla praticamente atingiram o mínimo metabólico aos 10 dias de omissão (Quadro 3) por apresentarem teores de Pi extremamente baixos. Tais espécies, mostraram queda na participação relativa de Pi em relação ao Pts de 56, 81 e 68\%, respectivamente, nas raízes basais, 10 dias após retirada do P da solução. As 
Quadro 2. Teores de fósforo total solúvel em ácido (Pts), fósforo inorgânico (Pi) e fósforo orgânico (Po) em folhas apicais e basais de cinco espécies de eucalipto após a omissão do nutriente (média de duas repetições)

\begin{tabular}{|c|c|c|c|c|c|c|c|}
\hline Folha & $\begin{array}{c}\text { Tempo de } \\
\text { omissão de P }\end{array}$ & $\begin{array}{l}\text { F ração } \\
\text { fosfatada }\end{array}$ & E. clöeziana & E. grandis & E. pellita & E.pilularis & E. urophylla \\
\hline & Dia & & 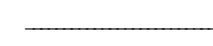 & $\mathrm{g}$ & -1 matéria fr & $\mathrm{ca}$ & \\
\hline \multirow[t]{3}{*}{ Apical } & 0 & $\begin{array}{l}\text { Pts } \\
\mathrm{Pi} \\
\mathrm{Po}\end{array}$ & $\begin{array}{l}55,66 \\
30,98(56)(1) \\
24,68(44)\end{array}$ & $\begin{array}{l}30,43 \\
11,94(39) \\
18,48(61)\end{array}$ & $\begin{array}{l}26,89 \\
12,69(47) \\
14,19(53)\end{array}$ & $\begin{array}{l}28,50 \\
13,15(46) \\
15,34(54)\end{array}$ & $\begin{array}{l}41,57 \\
22,04(53) \\
19,52(47)\end{array}$ \\
\hline & 10 & $\begin{array}{l}\text { Pts } \\
\mathrm{Pi} \\
\mathrm{Po}\end{array}$ & $\begin{array}{l}36,69 \\
7,29(20) \\
29,40(80)\end{array}$ & $\begin{array}{l}30,16 \\
4,37(14) \\
25,79(86)\end{array}$ & $\begin{array}{l}26,24 \\
4,01(15) \\
22,22(85)\end{array}$ & $\begin{array}{l}24,49 \\
5,87(24) \\
18,62(76)\end{array}$ & $\begin{array}{l}29,51 \\
6,03(20) \\
23,48(80)\end{array}$ \\
\hline & 20 & $\begin{array}{l}\mathrm{Pts} \\
\mathrm{Pi} \\
\mathrm{Po}\end{array}$ & $\begin{array}{l}30,30 \\
4,42(15) \\
25,87(85)\end{array}$ & $\begin{array}{l}13,64 \\
3,61(26) \\
10,03(74)\end{array}$ & $\begin{array}{l}12,80 \\
3,26(26) \\
9,53(74)\end{array}$ & $\begin{array}{l}11,52 \\
2,77(24) \\
8,74(76)\end{array}$ & $\begin{array}{l}15,29 \\
2,46(16) \\
12,82(84)\end{array}$ \\
\hline \multirow[t]{3}{*}{ Basal } & 0 & $\begin{array}{l}\text { Pts } \\
\mathrm{Pi} \\
\mathrm{Po}\end{array}$ & $\begin{array}{l}159,18 \\
41,02(26) \\
118,16(74)\end{array}$ & $\begin{array}{l}20,88 \\
9,63(46) \\
11,24(54)\end{array}$ & $\begin{array}{l}17,52 \\
9,38(53) \\
8,13(47)\end{array}$ & $\begin{array}{l}50,23 \\
48,26(96) \\
1,96(4)\end{array}$ & $\begin{array}{l}19,41 \\
10,44(54) \\
8,97(46)\end{array}$ \\
\hline & 10 & $\begin{array}{l}\text { Pts } \\
\mathrm{Pi} \\
\mathrm{Po}\end{array}$ & $\begin{array}{r}127,47 \\
22,16(17) \\
105,31(83)\end{array}$ & $\begin{array}{l}18,96 \\
3,29(17) \\
15,66(83)\end{array}$ & $\begin{array}{l}15,62 \\
3,72(24) \\
11,90(76)\end{array}$ & $\begin{array}{l}31,55 \\
19,00(60) \\
12,54(40)\end{array}$ & $\begin{array}{l}21,39 \\
4,71(22) \\
16,68(78)\end{array}$ \\
\hline & 20 & $\begin{array}{l}\mathrm{Pts} \\
\mathrm{Pi} \\
\mathrm{Po}\end{array}$ & $\begin{array}{l}47,82 \\
11,74(25) \\
36,08(75)\end{array}$ & $\begin{array}{l}15,74 \\
2,65(17) \\
13,08(83)\end{array}$ & $\begin{array}{l}12,42 \\
3,35(27) \\
9,06(73)\end{array}$ & $\begin{array}{l}18,89 \\
4,80(25) \\
14,09(75)\end{array}$ & $\begin{array}{l}9,23 \\
2,35(26) \\
6,87(74)\end{array}$ \\
\hline
\end{tabular}

(1) Número entre parênteses indica participação relativa (\%) de cada componente em relação ao fósforo total solúvel (Pts). DMS (5\%) Espécie x Tempo (fol has apicais): Pts = 5,54; Pi =2,49; Po = 5,83. CV (\%): 7,92; 5,75 e 8,98, respectivamente. DMS (5\%) Espécie $x$ Tempo (fol has basais): Pts =3,54; Pi =2,68; Po =4,51. CV (\%): 2,59; 5,86 e 4,98, respectivamente.

partes basais das plantas seriam, neste caso, fonte de $P$ para as partes apicais.

É importante observar que o principal reservatório deP deE. döeziana eE. pilularis foram as fol has vel has, enquanto, nas demais espécies, o reservatório de $\mathrm{P}$ foi maior nas fol has novas (Quadro 2).

Quanto às fol has inferiores, ressal ta-se o elevado teor de $\mathrm{P}$ no $\mathrm{E}$. pilularis e, principalmente, no $\mathrm{E}$. clöeziana no início da omissão do nutriente em relação às demais espécies. Especificamente no caso de $E$. clöeziana, o maior conteúdo do $P$ foi devido à participação do $\mathrm{Po}$ (74\%), enquanto, em E. pilularis, o Pi foi responsável por $96 \%$ do Pts. Sem dúvida, o maior conteúdo de P em E. clöeziana, observado ao ser cultivado em solo com el evado fornecimento de P (Furtini Neto et al., 1996), deveu-se ao acúmulo na forma de Po nas fol has mais vel has (Quadro 2).

O acúmulo de Pi por E. clöeziana sob elevado fornecimento de $\mathrm{P}$ foi compartimentalizado nas raízes (Quadro 3), chegando a partici pação do Pi em relação aos $\mathrm{Pts}$ a atingir $93 \%$ nas basais. Tais evidências revelam que $E$. clöeziana, diferentemente das demais espécies, armazena o excesso de Pi em níveis das raízes, especificamente em raízes menos ativas metabolicamente (Quadro 3). I sto concorda com as observações de Clarkson (1985) de que a maior retenção de Pi pelas raízes em condições de absorção limitada deveu-se ao fato de estarem mais próximas da fonte de suprimento.

Nas demais espécies, teores iniciais de Pi mais el evados em fol has e raízes apicais mostraram que os teci dos jovens, em formação e expansão, têm maior capacidade de armazenar Pi etorná-los drenos mais fortes para o $\mathrm{Pi}$ absorvido, em razão de sua maior atividade metabólica ou de sua maior taxa de transpiração. Nesse caso, as maiores concentrações citoplasmáticas de Pi ativam o mecanismor regulador em nível de tonoplasto, propiciando o seu armazenamento, ou seja, a regulação dos fluxos citoplasma-vacúolo e vacúol o-citoplasma, mediada por ajustes nas taxas de "turnover" ou na quantidade de carregadores ativos (Clarkson, 1985). Embora Bieleski (1973) afirme que, quando as folhas se tornam senescentes, ocorre grande perda líquida de fósforo, os resultados desteestudo indicaram queE. clöeziana não se adaptou a essas evidências ou que, pelo menos, atrasou em comparação com outras espécies quanto à remobilização do $\mathrm{Pi}$ para folhas metabolicamente mais ativas. 
Quadro 3. Teores de fósforo total solúvel em ácido (Pts), fósforo inorgânico (Pi) e fósforo orgânico (Po) em raízes apicais e basais de cinco espécies de eucalipto após a omissão do nutriente (média de duas repetições)

\begin{tabular}{|c|c|c|c|c|c|c|c|}
\hline Raiz & $\begin{array}{c}\text { Tempo de } \\
\text { omissão de P }\end{array}$ & $\begin{array}{l}\text { F ração } \\
\text { fosfatada }\end{array}$ & E. clöeziana & E. grandis & E. pellita & E. pilularis & E. urophylla \\
\hline & Dia & & 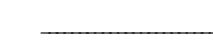 & - & $f^{-1}$ matéria $\mathrm{f}$ & $\mathrm{ca}$ & 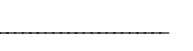 \\
\hline \multirow[t]{3}{*}{ Apical } & 0 & $\begin{array}{l}\text { Pts } \\
\mathrm{Pi} \\
\mathrm{Po}\end{array}$ & $\begin{array}{l}79,85 \\
67,46(85)^{(1)} \\
12,38(15)\end{array}$ & $\begin{array}{l}18,49 \\
9,07(49) \\
9,42(51)\end{array}$ & $\begin{array}{l}15,84 \\
8,85(56) \\
6,98(44)\end{array}$ & $\begin{array}{l}20,45 \\
14,67(72) \\
5,78(28)\end{array}$ & $\begin{array}{l}16,90 \\
12,31(73) \\
4,59(27)\end{array}$ \\
\hline & 10 & $\begin{array}{l}\text { Pts } \\
\mathrm{Pi} \\
\mathrm{Po}\end{array}$ & $\begin{array}{l}26,28 \\
11,09(42) \\
15,19(58)\end{array}$ & $\begin{array}{l}44,23 \\
21,73(49) \\
22,49(51)\end{array}$ & $\begin{array}{l}15,64 \\
2,89(19) \\
12,74(81)\end{array}$ & $\begin{array}{l}33,34 \\
11,35(34) \\
21,99(66)\end{array}$ & $\begin{array}{l}5,25 \\
1,35(26) \\
3,89(74)\end{array}$ \\
\hline & 20 & $\begin{array}{l}\text { Pts } \\
\mathrm{Pi} \\
\mathrm{Po}\end{array}$ & $\begin{array}{l}8,76 \\
2,43(28) \\
6,33(72)\end{array}$ & $\begin{array}{l}6,24 \\
1,38(22) \\
4,86(78)\end{array}$ & $\begin{array}{l}6,18 \\
1,37(22) \\
4,81(78)\end{array}$ & $\begin{array}{l}11,45 \\
3,73(33) \\
7,72(67)\end{array}$ & $\begin{array}{l}6,45 \\
1,41(22) \\
5,03(78)\end{array}$ \\
\hline \multirow[t]{3}{*}{ Basal } & 0 & $\begin{array}{l}\text { Pts } \\
\mathrm{Pi} \\
\mathrm{Po}\end{array}$ & $\begin{array}{l}43,75 \\
40,59(93) \\
3,15(7)\end{array}$ & $\begin{array}{l}13,46 \\
5,56(41) \\
7,93(59)\end{array}$ & $\begin{array}{l}17,53 \\
5,57(32) \\
11,96(68)\end{array}$ & $\begin{array}{l}26,72 \\
17,50(65) \\
9,22(35)\end{array}$ & $\begin{array}{l}11,16 \\
7,08(63) \\
4,08(37)\end{array}$ \\
\hline & 10 & $\begin{array}{l}\text { Pts } \\
\mathrm{Pi} \\
\mathrm{Po}\end{array}$ & $\begin{array}{l}37,29 \\
17,35(46) \\
19,93(54)\end{array}$ & $\begin{array}{l}11,48 \\
2,43(21) \\
9,04(79)\end{array}$ & $\begin{array}{l}5,93 \\
1,07(18) \\
4,86(82)\end{array}$ & $\begin{array}{l}29,10 \\
7,68(26) \\
21,42(74)\end{array}$ & $\begin{array}{l}14,46 \\
2,23(15) \\
12,23(85)\end{array}$ \\
\hline & 20 & $\begin{array}{l}\text { Pts } \\
\mathrm{Pi} \\
\mathrm{Po}\end{array}$ & $\begin{array}{l}10,31 \\
5,90(57) \\
4,40(43)\end{array}$ & $\begin{array}{l}6,42 \\
1,88(29) \\
4,54(71)\end{array}$ & $\begin{array}{l}6,84 \\
1,90(28) \\
4,94(72)\end{array}$ & $\begin{array}{l}15,77 \\
5,90(37) \\
9,87(63)\end{array}$ & $\begin{array}{l}4,67 \\
1,31(28) \\
3,36(72)\end{array}$ \\
\hline
\end{tabular}

(1) Número entre parênteses indica participação relativa (\%) de cada componente em relação ao fósforo total solúvel (Pts). DMS (5\%) Espécie x Tempo (raízes apicais): Pts =2,36; Pi =1,78; Po =4,51. CV(\%): 3,22; 4,47 e 9,35, respectivamente. DMS (5\%) Espécie x Tempo (raízes basais): Pts =3,38; Pi =0,91; Po =3,71. CV (\%): 5,70; 3,14 e 12,18, respectivamente.

E. grandis e E. pellita mostraram padrão mais homogêneo de comportamento. A participação relativa do Po em relação ao Pts aumenta com o período de omissão, al iado à redução da participação relativa de $\mathrm{Pi}$, indicando que essas espécies mobilizam sua reserva dePi para manter seu crescimento. Também os compartimentos mais ativos da planta (fol has e raízes novas) apresentaram teores mais el evados de fósforo, confirmando observações de Biel eski (1973) sobrea perda líquida de fósforo deórgãos senescentes. Tal comportamento teria como reflexo final a maior produção de biomassa por essas espécies.

É importanteressaltar queE. grandiseE. pellita, em praticamente todos os compartimentos analisados, atingiram teores bastante baixos de $\mathrm{Pi}$, 10 dias após a retirada do P da solução, indicativo de um menor "poder tampão interno de $\mathrm{Pi}$ "e, ou, do maior crescimento dessas espécies em comparação às demais. Tal comportamento evidencia que, após 10 dias sem suprimento de $\mathrm{P}$, as plantas dessas espécies já poderiam apresentar problemas, enquanto as de outras espécies suportariam a falta deP por mais tempo. Sem dúvida, essas observações devem ser consideradas na adaptação das espécies às condições nutricional mente distintas de sol o.

\section{CONCLUSÕES}

1. As espécies deeucal ipto estudadas apresentaram comportamento distinto quanto às frações fosfatadas. E. clöeziana apresentou maior conteúdo de fósforo total solúvel (Pts) queas demais espécies, indicando uma taxa relativamentealta de absorção de fósforo.

2. A queda no conteúdo de Pts após 20 dias de omissão de fósforo indicou que E. clöeziana e E. urophyilla suportaram por mais tempo a interrupção no fornecimento de fósforo, quando bem nutridas, sem paralisarem o crescimento.

3. E. grandis e E. pellita apresentaram teores bastante baixos de Pi, após 10 dias de omissão de fósforo, evidenciando que tais espécies, com poucos dias sem suprimento de fósforo, poderiam apresentar redução no crescimento.

\section{LITERATURA CITADA}

BARROS, N.F.; NOVAIS, R.F.; CARDOSO, J R. \& MACEDO, P.R.O. Algumas relações solo-espécies de eucalipto em suas condições naturais. In: BARROS, N.F. \& NOVAIS, R.F., eds. Relação solo-eucalipto. Viçosa, Folha de Viçosa, 1990. p.124. 
BIELESKI, R.L. \& FERGUSON, I.B. Physiology and metabolism of phosphate and its compounds. In: LAUCHLI, A. \& BIELESKI, R.L., eds. Enciclopedia of plant physiology: Inorganic plant nutrition. Berlin, Springer-Verlag, 1983. p.422-429.

BIELESKI, R.L. Phosphate pools, phosphate transport, and phosphate availability. Ann. Rev. Plant Physiol., 24:225-252, 1973.

BRAGA, J.M. \& DEFELIPO, B.V. Determinação espectrofotométrica de fósforo em extratos de solos e plantas. R. Ceres, 21:73-85, 1974.

CHAPIN, F.S.; FOLLETT, J.M. \& O'CONNOR, K.F. Growth, phosphate absorption and phosphorus chemical fractions in two Chionochlos species. J. Ecol., 70:305-321, 1982.

CLARK, R.B. Characterization of phosphate of intact maizeroots. J. Agric. Food. Chem., 23:458-460, 1975.

CLARKSON, D.T. Factors affecting mineral nutrient acquisition by plants. Ann. Rev. Plant Physiol., 36:77-115, 1985.

EPSTEIN, E. \& J EFFRIES, R.L. The genetic basics of selective ion transport in plants. Ann. Rev. Plant Physiol., 15:169184, 1964.

FABRES, A.S. Disponi bilidade de fósforo em sol os e concentrações críticas de diferentes frações de fósforo em plantas dealface cultivadas em amostras de diferentes solos. Viçosa, Universidade Federal de Viçosa, 1986. 39p. (Tese de Mestrado)
FURTINI NETO, A.E.; BARROS, N.F.; GODOY, M.F. \& NOVAIS, R.F. Eficiência nutricional, de mudas de Eucalyptus em relação a fósforo. R. Árv., 20:17-28, 1996.

GERLOFF, G.C. \& GABELMAN, W.H. Genetic basics of inorganic plant nutrition. In: LAUCHLI, A \& BIELESKI, R.L., eds. Inorganic plant nutrition. Berlin, Spring Verlag, 1983. p.453480.

GLASS, A.D.M. \& SIDDIQI, M.Y.The control of nutrient uptake rates in relation to the inorganic composition of plants. In: TINKER, P.B. \& LAUCHLI, A., eds. Advances in plant nutrition. New York, Praeger Publishers, 1984. p.103-147.

HOGUE, E.; WILCOX, G.E. \& CANTLIFFE, D.J . Effect of soil phosphorus levels on phosphate fractions in tomato leaves. J. Am. Soc. Hortic. Sci., 95:174-176, 1970.

LEE, R.B. \& RATCLIFFE, R.G. Phosphorus nutrition and the intracelular distribution of inorganic phosphate in pea root tips: a quantitative study using 31P - NMR. J . Exper. Bot., 34:1222-1224, 1983.

MARTINEZ, H.E.P. Cinética da absorção e das frações fosfatadas em sete variedades de soja (Glycinemax, L. Merril). Viçosa, Universidade Federal de Viçosa, 1992. Não paginado. (Relatório Final de Programa de Pós-Doutoramento)

SMILLIE, R.M. \& KROTKOV, F. The estimation of nucleic acids in some algae and higher plants. Can. J. Bot., 38:31-49, 1960.

VOSE, P.B. Genetical aspects of mineral nutrition - Progress to date. In: GABELMAN, H.W. \& LOUGWMAN, B.C., eds. Genetic aspects of plant mineral nutrition. Dordrecht, Martinus Nyhoff, 1987. p.3-13. 